at precisely the same frequency and the acoustoelectric current is measured as the relative phase is increased from 0 to $360^{\circ}$. The current (and also the energy in the standing wave) reach a maximum near $180^{\circ}$ : at this point a peak travelling in one direction and a trough travelling in the other collide, and the electrons in the 2DEG are caught in a terrific storm. If the amplitudes of the waves are large enough, the response of the electrons is no longer purely harmonic, which is a clear indication that an acoustic shock wave is travelling through the nanobridge.

This combination of nanomechanics, two-dimensional electronics and some very extreme conditions could scarcely have been imagined a few years ago. Nanomechanical devices will no doubt continue to push further into the quantum world, teaching us more about the interactions between electrons and phonons, and acting as a bridge into the unknown.

\section{References}

1. Beil, F. W. et al. Phys. Rev. Lett. 100, 026801 (2008).

2. Singleton, J. Band Theory and Electronic Properties of Solids (Oxford Univ. Press, Oxford, 2001)

3. Martin, J., Cichos, F., Huisken, F. \& von Borczskowski, C. Nano Lett. 8, 656-660 (2008).

4. Abedin, M. N., Strashilov, V. L. \& Das, P. Solid State Electron. 33, 65-68 (1990).

\title{
Quantum drums beat as one
}

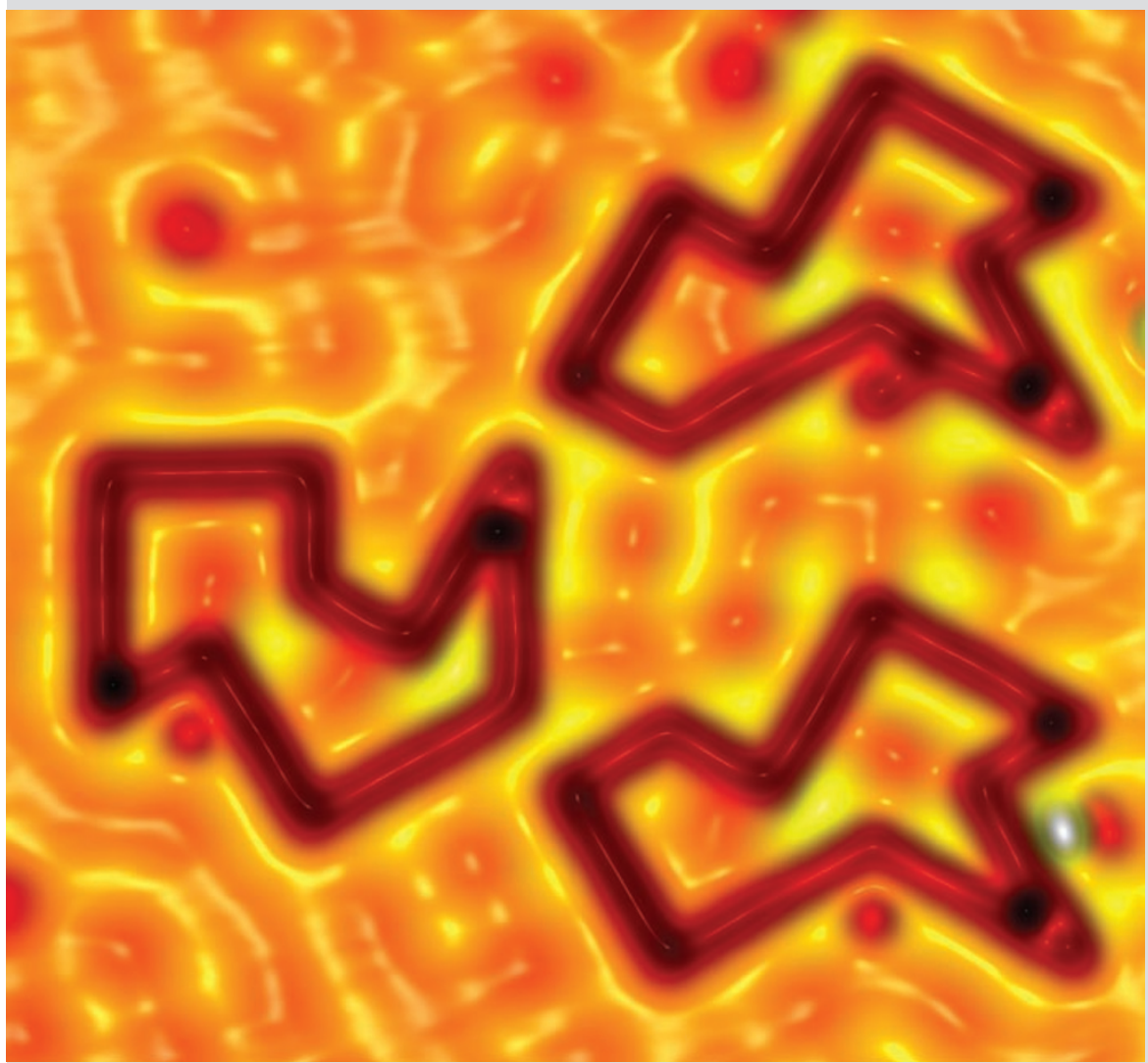

First there was "IBM" written with 35 xenon atoms on a nickel surface. The quantum corral came next, followed by the quantum mirage, and now, pictured above, the quantum drum has made its debut. The ability of the scanning tunnelling microscope (STM) to manipulate and probe matter at the level of single atoms and molecules has been established for almost two decades, but physicists can always find new tricks to perform, as Hari Manoharan and co-workers at Stanford University now demonstrate by determining the quantum phase of the wave function of a nanostructure without recourse to interferometry (Science 319, 782-787; 2008).

Manoharan and co-workers begin by using a homemade STM operating at $4.2 \mathrm{~K}$ to move carbon monoxide molecules around a copper surface to make three different nine-sided figures known as the Bilby (left), Hawk (bottom) and Broken Hawk (top). All three figures are composed of seven identical triangles that are arranged so that the nanostructures have the same area and perimeter. Moreover, the Bilby and the Hawk shapes are designed so that they are isospectral - in other words, they both have the same electronic structure even though their shapes are different. In the image, which measures $15 \mathrm{~nm}$ across, each shape is made from 45 molecules and contains six electrons.

The Stanford team then uses the STM to probe the wave function of the electrons confined within the nanostructures. Normally it is only possible to determine the amplitude of the wave function with this approach, but Manoharan and co-workers exploit the isospectral properties of the shapes to determine the phase as well. The team also repeat this feat with more complicated shapes called the Ayeaye and the Beluga that are made of 21 identical triangles each. So far the technique only works on shapes that have isospectral partners, but a large number of such shapes are known. Further details, images and movies are available at http://mota.stanford.edu/.

Quantum phases can also be measured with interferometric techniques. However, these experiments can be complicated because they require both a reference beam and an object beam to make the measurement.

The name quantum drum is a reference to the mathematician Mark Kac who, in 1966, asked: "Can one hear the shape of a drum?" Experiments on systems ranging from microwaves to liquid crystals have since confirmed that, for example, metal cavities can have matching resonant frequencies even if they do not have the same shape. In other words, drums with different shapes can sound the same, so the answer to Kac's question is "No". The Stanford team has now exploited this fact in its method for extracting quantum phases.

Peter Rodgers 